\title{
Ischemic stroke associated with COVID-19: a systematic review and meta-analysis
}

\author{
Wenzhang Luo ${ }^{1}$. Xiang Liu ${ }^{1} \cdot$ Kunyang Bao ${ }^{1}$. Changren Huang ${ }^{1,2,3,4}$
}

Received: 2 July 2021 / Revised: 1 October 2021 / Accepted: 5 October 2021 / Published online: 15 October 2021

(c) The Author(s), under exclusive licence to Springer-Verlag GmbH Germany 2021

\begin{abstract}
Background Coronavirus disease 2019 (COVID-19), a contagious infectious disease caused by severe acute respiratory syndrome coronavirus 2 (SARS-CoV-2), has spread across the world. Apart from respiratory complications, an increasing number of patients with ischemic stroke have been reporting.

Objective This systematic review and meta-analysis aims to explore the characteristics of ischemic stroke after SARS-CoV-2 infection, and provides valuable reference materials for subsequent clinical treatment.

Materials and methods PubMed, Web of Science, and Ovid-Embase databases were searched up to 24th March 2021. We utilized the search strategy of medical subject headings combined with entry terms to search all related literatures. All studies identified with the electronic and manual searches were listed by citation, title, authors, and abstract. Only studies involving patients with COVID-19-related stroke were eligible. The references of included studies were also manually screened.

Results The meta-analysis was conducted following the PRISMA and MOOSE reporting guidelines. Bias risk was assessed using the Newcastle-Ottawa Scale (NOS). Ten articles, including 26,691 participants and 280 patients with ischemic stroke and COVID-19, were selected. The pooled prevalence of ischemic stroke in COVID-19 was 2\% (95\% CI 1-2\%; $p<0.01$ ). The pooled proportions of hypertension, hyperlipidemia and diabetes in COVID-19-related ischemic stroke was $66 \%$ (95\% CI $51-81 \% ; p<0.01$ ), $48 \%$ (95\% CI 19-76\%; $p<0.01$ ) and 40\% (95\% CI 29-51\%; $p<0.01$ ), respectively. Notably, the pooled proportions of female was $36 \%(95 \%$ CI $21-50 \% ; p<0.01)$ in patients with COVID-19 and stroke. In addition, in TOAST classification, cryptogenic stroke subtype was associated with a high trend, and its pooled proportion was $35 \%$ (95\% CI $12-59 \% ; p<0.01)$.

Conclusion Ischemic stroke caused by COVID-19 has its own unique clinical features. Although common high-risk factors can also be observed, its importance may have changed. The major inflammatory storm of COVID-19 is more likely to occur in male patients. The increase in the proportion of cryptogenic stroke has also made stroke related to COVID-19 complicated.
\end{abstract}

Keywords Ischemic stroke $\cdot$ COVID-19 $\cdot$ SARS-CoV-2 $\cdot$ Clinical characteristics

Changren Huang

changrenhuang1@swmu.edu.cn

1 Department of Neurosurgery, The Affiliated Hospital of Southwest Medical University, No.25 of Taiping Street, Luzhou 646000, Sichuan, China

2 Neurosurgical Clinical Research Center of Sichuan Province, Luzhou, China

3 Academician (Expert) Workstation of Sichuan Province, The Affiliated Hospital of Southwest Medical University, Luzhou, China

4 Laboratory of Neurological Diseases and Brain Functions, The Affiliated Hospital of Southwest Medical University, Luzhou, China

\section{Introduction}

The clinical manifestations of patients with new coronary pneumonia vary greatly from asymptomatic infection to severe pneumonia that may cause respiratory failure and death [1]. In an early study in Wuhan, China, 2.3\% of 214 patients hospitalized with COVID-19 suffered an ischemic stroke [2]. Several other studies had also reported that they have found ischemic stroke in patients with COVID-19, which was comparable to the rate found in Wuhan, China in the early stages of the pandemic [3-5]. However, more recently, a large cohort study from New York reported ischemic stroke only in $0.9 \%$ of 3556 hospitalized patients 
with COVID-19 [6]. The incidence rates vary greatly among different research cohorts.

Although the cause of ischemic stroke associated with COVID-19 is unclear, previous studies have hypothesized that inflammatory cytokine storms may be a trigger for hypercoagulable state or endothelial damage [7]. At the same time, several studies have described the different mechanisms by which SARS-CoV-2 can cause neurological disorders and stroke. Many of these mechanisms focus on angiotensin-converting enzyme-2 (ACE-2), the binding site of SARS-CoV-2, and its function as a trigger for a series of events leading to vasoconstriction, hypertension, or thrombosis imbalance. Other studies have suggested that immune-mediated mechanisms and over expression of cytokines, hypercoagulable state and thromboembolism are potential causes of stroke [8-11]. It can be observed that the COVID-19 is closely related to ischemic stroke, because it has potential factors leading to stroke.

There is imperative to understand stroke demographic and clinical features since stroke remains an emergency. This article serves as a systematic review and meta-analysis of relevant literatures to summarize the characteristics of ischemic stroke associated with COVID-19 and discusses its value for clinical treatment.

\section{Methods}

This systematic review follows the PRISMA (Preferred Reporting Items for Systematic Reviews and Meta-Analyses) guidelines statement $[12,13]$. The PRISMA flow diagram of the selection process and the MOOSE checklist are provided in Fig. 1.

\section{Data source and strategy}

We searched PubMed, Web of Science, and Ovid-Embase to identify relevant studies. We used search terms related to COVID-19 and stroke. Detailed search strategies are presented in supplemental document 1 .

\section{Eligibility criteria and study selection}

For the selection of the papers, the following inclusion criteria were defined: (1) articles focused on ischemic stroke associated with COVID-19, (2) articles with original data (e.g., cohort, retrospective, case-control studies), (3) diagnosis of all patients with COVID-19 was based on positive real-time polymerase chain reaction (PCR) assay for severe acute respiratory syndrome coronavirus 2 , (4) diagnosis of all patients with stroke was based on imaging and clinical symptoms, (5) articles published in English, and (6) objects of research $>10$ patients. We excluded studies

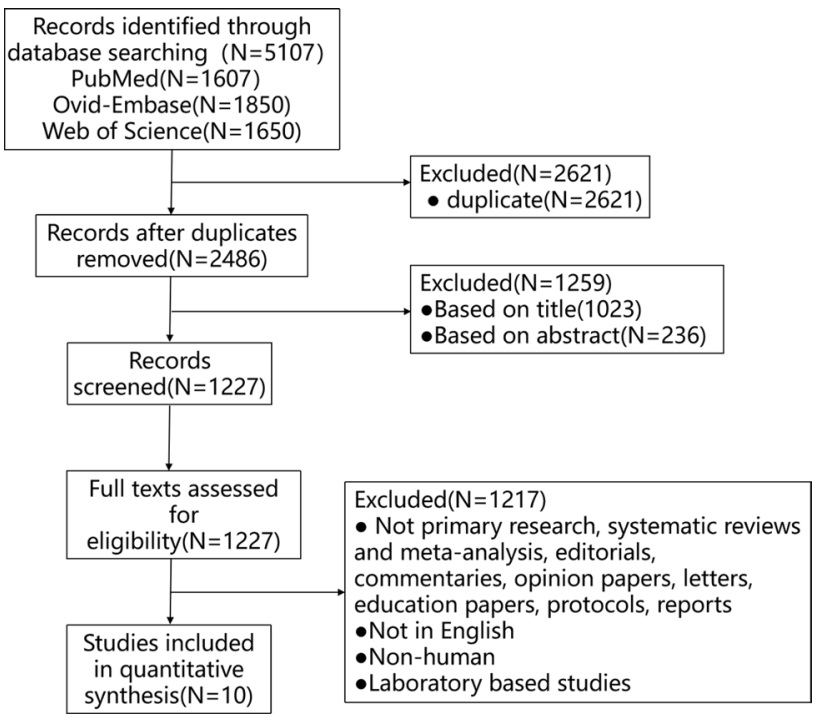

Fig. 1 Flow diagram

such as not primary research, systematic reviews and meta-analysis, editorials, commentaries, opinion papers, letters, education papers, protocols, reports, not in English, non-human and laboratory-based studies.

\section{Data extraction}

Two independent reviewers (Wenzhang Luo and Xiang Liu) screened the titles and abstracts according to the selection criteria. Three independent reviewers (Wenzhang Luo, Xiang Liu, and Kunyang Bao) screened the full texts. Data were extracted independently by two reviewers (Wenzhang Luo and Kunyang Bao), and any disagreements were resolved through consensus. We performed data extraction using a predefined form including the following data: first author name, country, journal, sample size, number of ischemic stroke in the study, mean age and list of the outcomes of interest.

During the data extraction, the outcomes of interest were classified into five groups: gender, number of patients with hypertension, number of patients with hyperlipidemia, number of patients with diabetes and TOAST classification (Supplemental Document 2).

\section{Assessment of risk of bias}

The bias risk assessment was carried out using the Newcastle-Ottawa Scale (NOS) by two authors (Wenzhang Luo and Kunyang Bao) [14]. Another author (Changren Huang) adjudicated in case consensus was not reached. 


\section{Data analysis}

The meta-analysis was performed with $\mathrm{R} \times 644.0 .3$ and RStudio using the 'Matrix', 'Meta' and 'Metafor' packages and led to pooled proportions with $95 \%$ confidence intervals (95\% CIs).

The $I^{2}$ was used to assess heterogeneity. Low, moderate and high levels of heterogeneity were defined by $I^{2}$ values of $25 \%, 50 \%$, and $75 \%$, respectively. The data which had low heterogeneity chose the fixed-effects meta-analysis and others chose random-effects meta-analysis [15]. All analyses were done using 2 -tailed tests with a $p$ value $<0.05$ considered statistically significant.

\section{Results}

\section{Study selection and characteristics}

The searches in PubMed, Web of Science, and Ovid-Embase retrieved 5107 citations. Following removal of duplicates and screening of titles and abstracts, 1227 articles were selected for full-text evaluation. Ten articles were retained after full-text assessment. The included studies were from China, United States of America, United Arab Emirates, Switzerland, Canada, Brazil, Greece, Italy, Finland, Turkey, Lebanon, Iran, India, and New Zealand. The studies involved a total of 26,691 patients.

In one paper, it only included COVID-19 patients with stroke, and did not mention the overall number of COVID19 infection [16]. In addition, in another paper, though it included 17,799 participants, 6200 patients without stroke were received in details. Other centers provided summary data that could not be used for comparison [17].

The reported mean age ranged from 48.1 to 75.7 years, and $35.1 \%$ ( 52 of $148 ; 8$ studies) of the patients were females (Table 1).

\section{Risk of bias of included study}

The bias risk assessment showed that, among the included papers, nine respected the criteria for a fair-quality study, only one is poor quality (Supplemental Document 3).

\section{Synthesis of results}

\section{Gender}

The forest plot of gender shows that the pooled proportions of female were $36 \%$ (95\% CI $21-50 \% ; p<0.01 ; I^{2}=77 \%$; random-effects model; Fig. 2). It suggests that stroke related to COVID-19 is more common in men. Especially in John et al. study, male accounted for 95\% (18 of 19) [18].

\section{Hypertension}

We identified 280 hospitalized patients with ischemic stroke associated with COVID-19. 165 of those had hypertension. The reported prevalence of hypertension ranged from 37 to $95 \%$, and the pooled prevalence was $66 \%$ (95\% CI 51-81\%; $p<0.01 ; I^{2}=88 \%$; random-effects model; Fig. 3). Hypertension continues to be a risk factor for stroke. However, patients with COVID-19 and stroke were less likely to have hypertension when compared with historical stroke controls in Yaghi et al. study [6].

Table 1 Characteristics of included studies

\begin{tabular}{|c|c|c|c|c|c|c|}
\hline \multirow[t]{2}{*}{ Reference, year } & \multirow[t]{2}{*}{ Countries } & \multirow[t]{2}{*}{ Journal } & \multirow[t]{2}{*}{ Sample size } & \multirow{2}{*}{$\begin{array}{l}\text { Ischemic } \\
\text { stroke, } \mathrm{n} \\
(\%)\end{array}$} & \multicolumn{2}{|l|}{ Ischemic stroke } \\
\hline & & & & & Mean age (SD) & Female, $n(\%)$ \\
\hline Li et al. 27 & China & Stroke Vasc Neurol & 219 & $10(4.6)$ & $75.7(10.8)$ & $5(50)$ \\
\hline Rothstein et al. 3 & USA & Stroke & 844 & $20(2.4)$ & $64(12)$ & $8(40)$ \\
\hline Grewal et al. 2020 & USA & Front Neurol & 650 & $13(2.0)$ & $61.6(\mathrm{NR})$ & $7(53.8)$ \\
\hline John et al. 18 & UAE & Clin Neurol Neurosurg & 591 & $19(3.2)$ & $48.1(10.8)$ & $1(5.3)$ \\
\hline Chen et al. 21 & China & Aging & 2037 & $10(0.5)$ & $74.1(12.8)$ & $5(50)$ \\
\hline Mendes et al. 18 & Switzerland & BMC Geriatr & 265 & $9(3.4)$ & NR & NR \\
\hline Shahjouei et al. 17 & $\begin{array}{l}\text { USA, Canada, Brazil, Greece, Italy, } \\
\text { Finland, Turkey, Lebanon, Iran, } \\
\text { India, New Zealand }\end{array}$ & EBioMedicine & 17,799 & $123(0.7)$ & NR & NR \\
\hline Yaghi et al. 6 & USA & Stroke & 3556 & $32(0.9)$ & NR & $9(28.1)$ \\
\hline Bach et al. 2020 & USA & Stroke Vasc Neurol & 683 & $20(2.9)$ & $63.1(10.7)$ & $6(30)$ \\
\hline Behzadnia et al. 16 & Iran & Ro J Neurol & $47 *$ & $24(51.1)$ & $73.1(11.8)$ & $11(45.8)$ \\
\hline
\end{tabular}

USA United States of America, UAE United Arab Emirates, NR not reported

*This study only included COVID-19 patients with stroke, and did not mention the overall number of COVID-19 infection 


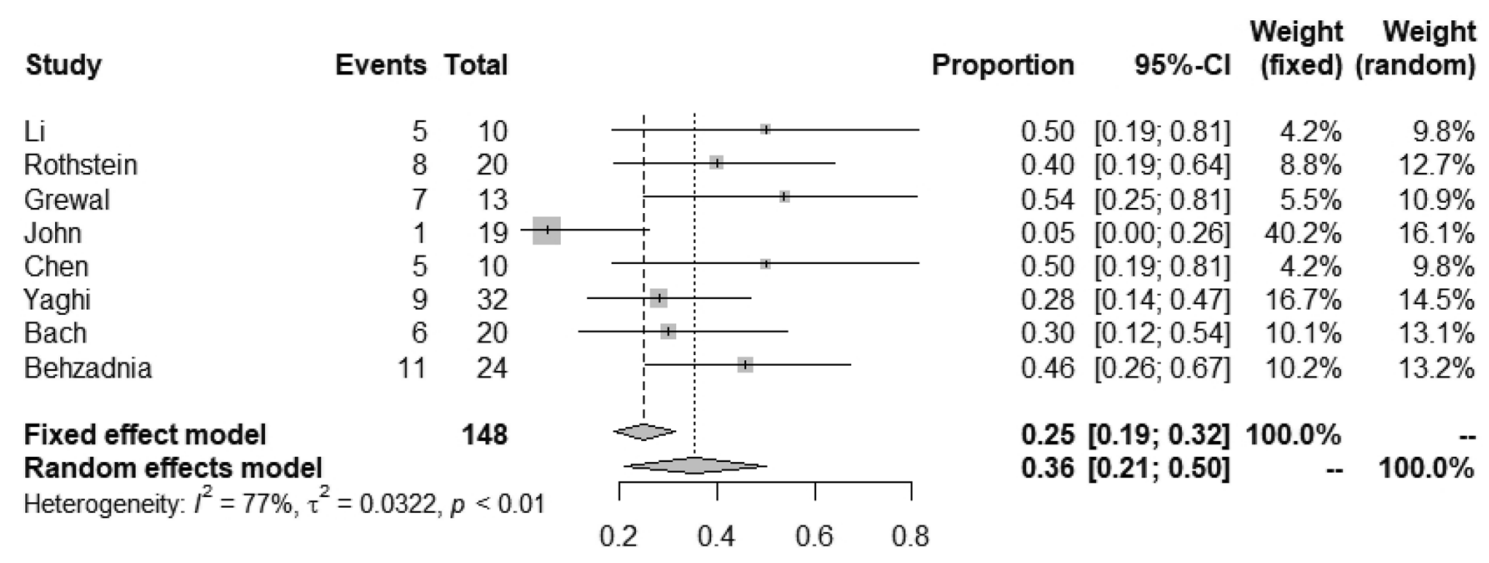

Fig. 2 Forest plot of gender proportion among hospitalized patients with ischemic stroke and COVID-19

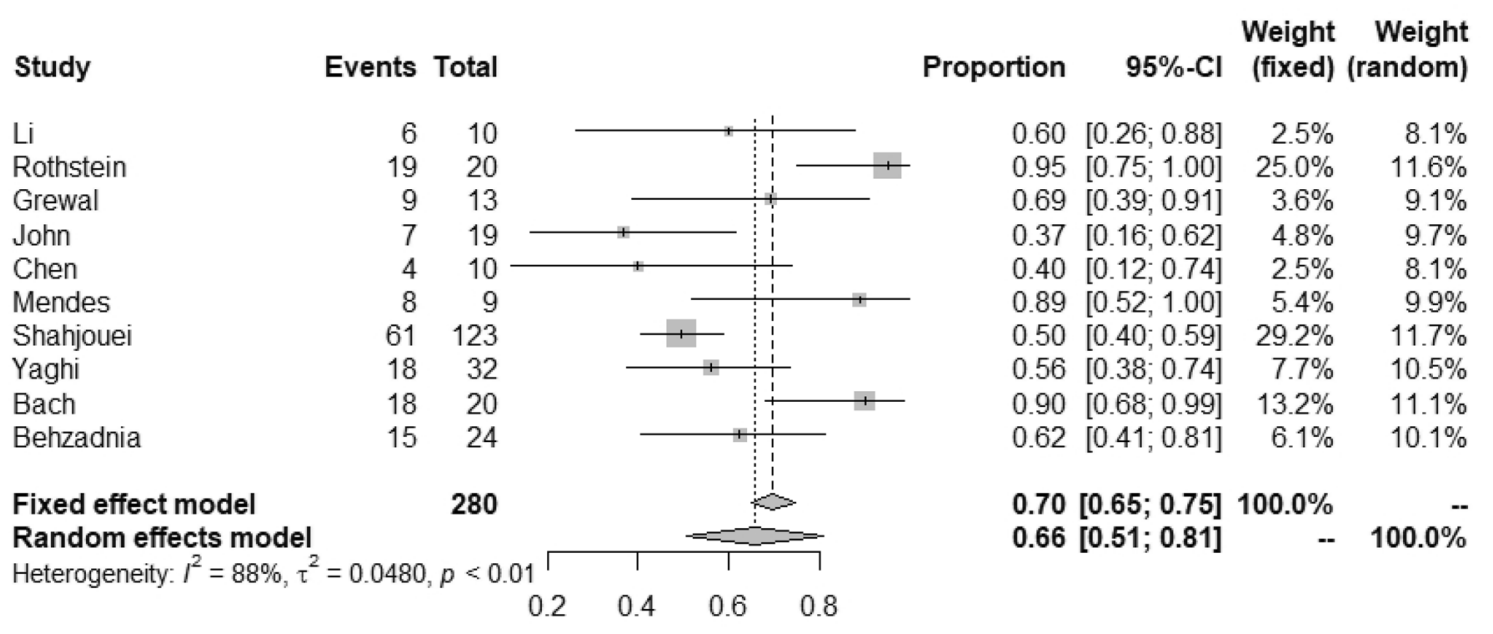

Fig. 3 Forest plot of hypertension prevalence among hospitalized patients with ischemic stroke and COVID-19

\section{Diabetes}

The reported prevalence of hypertension ranged from 20 to $65 \%$, and the pooled prevalence was $40 \%$ (95\% CI $29-51 \%$; $p<0.01 ; I^{2}=69 \%$; random-effects model; Fig. 4). Patients with diabetes mellitus in COVID-19-related stroke still account for a considerable proportion.

\section{Hyperlipidemia}

The reported prevalence of hyperlipidemia ranged from 5 to $80 \%$, and the pooled prevalence was $48 \%$ (95\% CI 19-76\%; $p<0.01 ; I^{2}=93 \%$; random-effects model; Fig. 5). In Mendes et al. study, there were no differences regarding other cerebrovascular risk factors, except for dyslipidemia, which was more frequent in stroke patients [19].

\section{TOAST classification for COVID-19-related stroke patients}

The TOAST classification denotes five subtypes of ischemic stroke: (1) large vessel disease, (2) small vessel disease, (3) cardioembolic, (4) cryptogenic, and (5) other defined mechanisms [20, 21]. The Table 2 shows the TOAST classification of our included studies. In our study, we found that the proportion of cryptogenic stroke was prominent. Its pooled prevalence was 35\% (95\% CI $12-59 \% ; p<0.01 ; I^{2}=89 \%$; random-effects model; Fig. 6). When compared with historical stroke controls, patients with COVID-19-related stroke were more likely to have a cryptogenic stroke subtype in Yaghi et al. study $(66 \%$; 95\% CI 47-81\%; $p<0.01)$ [6]. However, the incidence of stroke due to small vessel disease was low. Its pooled prevalence was $2 \%$ (95\% CI 0-5\%; $p=0.44 ; I^{2}=0 \%$; random-effects model; Fig. 7). 


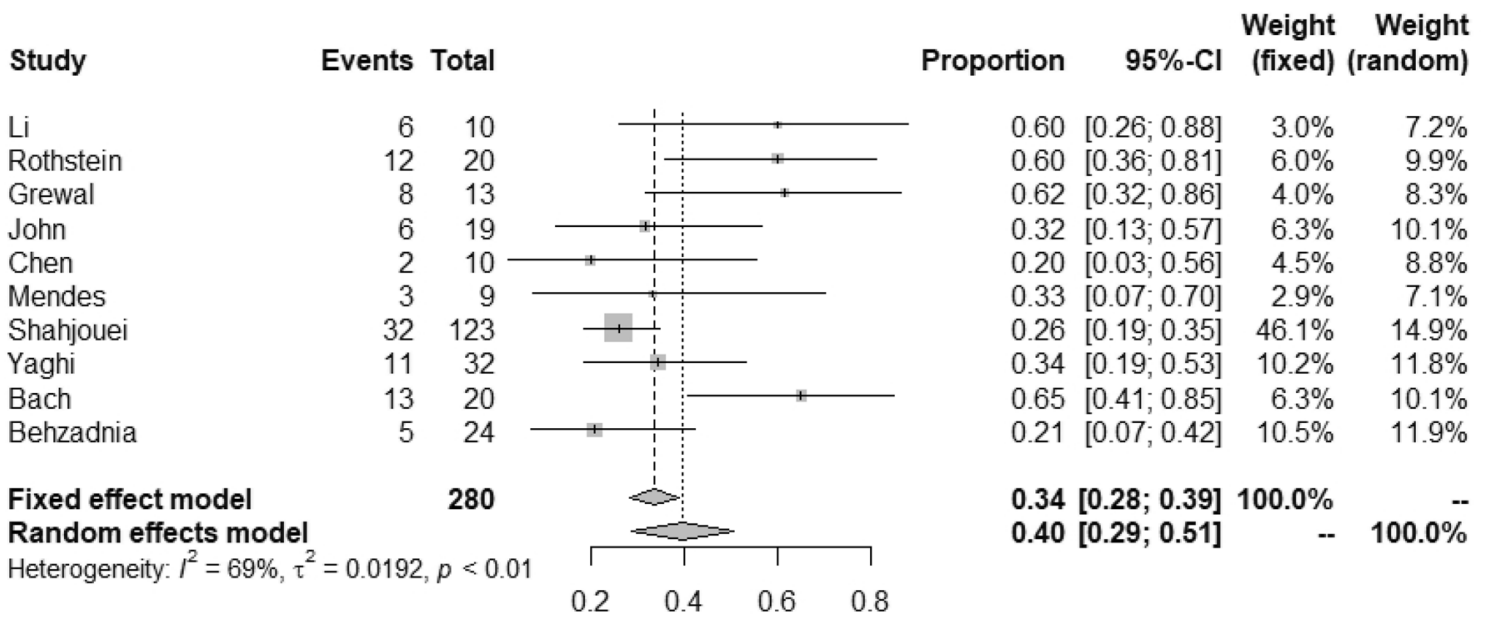

Fig. 4 Forest plot of diabetes prevalence among hospitalized patients with ischemic stroke and COVID-19

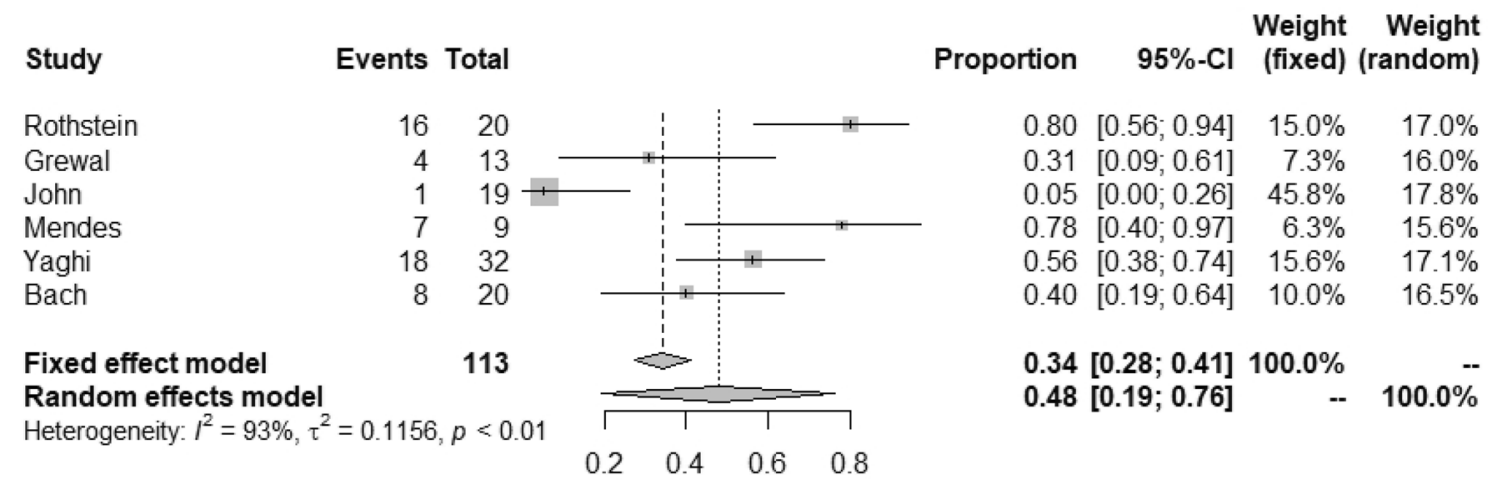

Fig. 5 Forest plot of hyperlipidemia prevalence among hospitalized patients with ischemic stroke and COVID-19

Table 2 TOAST classification of included studies

\begin{tabular}{llllll}
\hline Study & $\begin{array}{l}\text { Large vessel } \\
\text { disease }\end{array}$ & $\begin{array}{l}\text { Small vessel } \\
\text { disease }\end{array}$ & Cardioembolic & Cryptogenic & $\begin{array}{l}\text { Other } \\
\text { defined } \\
\text { mechanisms }\end{array}$ \\
\hline Li et al. 27 & 5 & 2 & 3 & 0 & 0 \\
Rothstein et al. 3 & 0 & 1 & 8 & 7 & 4 \\
Grewal et al. 2020 & 2 & 1 & 3 & 7 & 0 \\
John et al. 18 & 6 & 0 & 4 & 8 & 1 \\
Chen et al. 21 & NR & NR & NR & NR & NR \\
Mendes et al. 18 & NR & NR & NR & NR & NR \\
Shahjouei et al. 17 & NR & NR & NR & NR & NR \\
Yaghi et al. 6 & 2 & 0 & 7 & 21 & 2 \\
Bach et al. 2020 & 5 & 1 & 2 & 2 & 1 \\
Behzadnia et al. 16 & NR & NR & NR & NR & NR \\
\hline
\end{tabular}

$N R$ not reported 


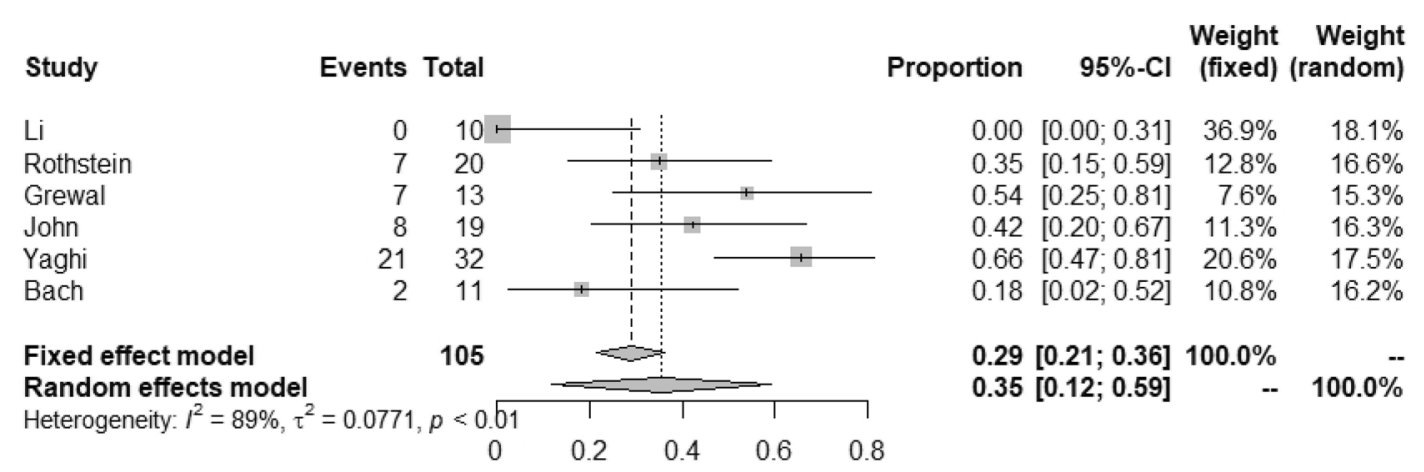

Fig. 6 Forest plot of cryptogenic stroke prevalence among hospitalized patients with ischemic stroke and COVID-19

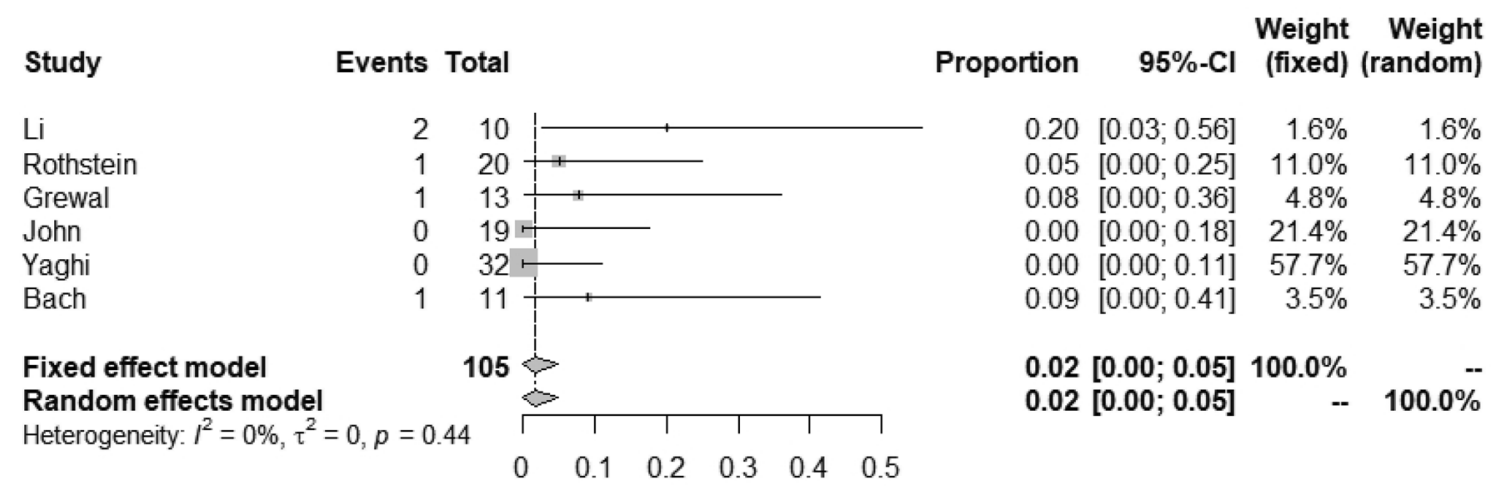

Fig. 7 Forest plot of small vessel disease stroke prevalence among hospitalized patients with ischemic stroke and COVID-19

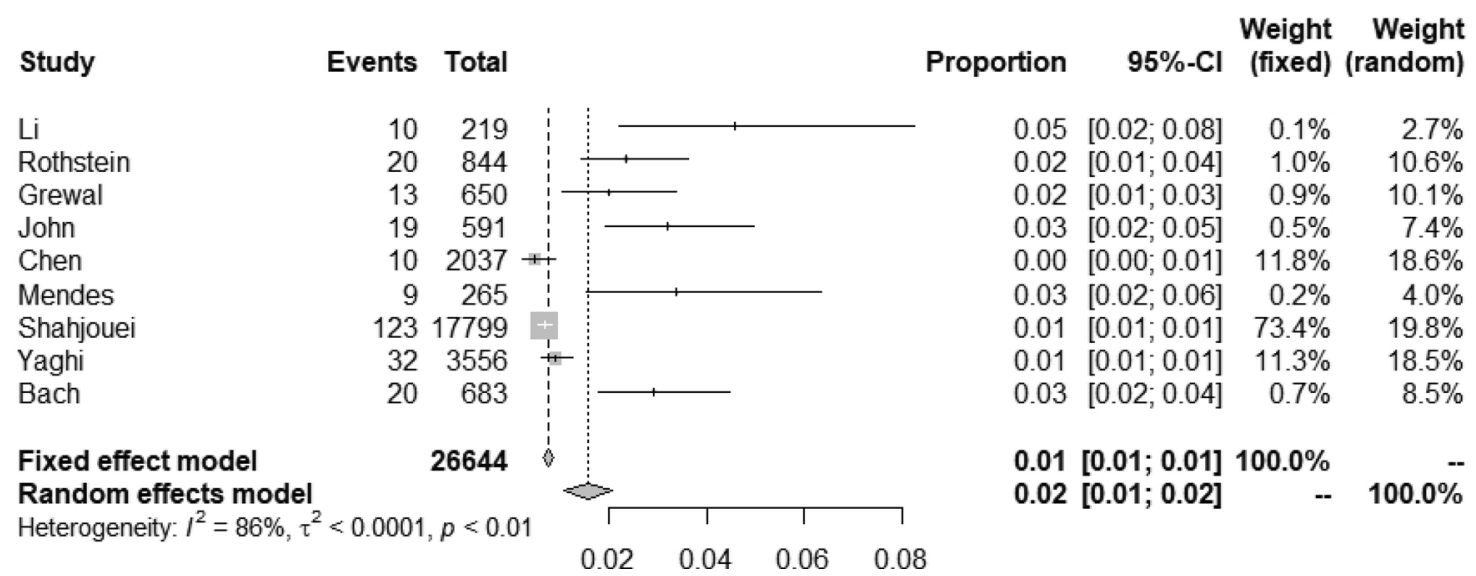

Fig. 8 Forest plot of the morbidity of stroke in COVID-19

\section{The morbidity of stroke in COVID-19 patient}

In our included studies, the morbidity of stroke in COVID-

19 patient ranged from 0 to $5 \%$. The pooled prevalence of ischemic stroke in COVID-19 was 2\% (95\% CI 1-2\%; $p<0.01 ; I^{2}=86 \%$; random-effects model; Fig. 8). Similarly, a recent systematic review polled 135 cases of ischemic 
stroke in patients with COVID-19, showing a stroke incidence varying from 0.9 to $2.7 \%$ [22].

\section{Discussion}

In this systematic review and meta-analysis, based on data from 10 relevant literature and 26,691 COVID-19 patients across all ages, we found that approximately $2 \%$ patients with COVID-19 infection could present with ischemic stroke. These data are similar to previous studies [2-5]. However, a study from a multinational cohort showed that hospitalized patients with SARS-CoV-2 infection indicated an overall stroke risk of $0.5 \%$ (pooled risk: $0.9 \%$ ) [17] Coincidentally, a large cohort study from New York reported ischemic stroke only in $0.9 \%$ of 3556 hospitalized patients with COVID-19 [6]. The incidence rate varies greatly between different research cohorts. Reasons for the difference are unknown, possibly be related to COVID-19 infection severity of hospitalized patients, prevalence of vascular risk factors in the population, ability to accurately diagnose all strokes in a situation of medical services being overwhelmed, and methodological differences. For instance, the ischemic stroke cohort in Mendes et al. study was all old patients whereas in John et al. study the ischemic stroke cohort mean age was 48.1 years.

Previous studies have shown that recent bacterial and/or viral infections may be triggers of acute ischemic stroke and may be related to the prothrombotic effects of inflammatory reactions [23]. SARS-CoV-2 has a spike protein surface unit that highly binds to human ACE-2 receptor. It may cause endothelial cell apoptosis and neuronal damage [24]. Viral infection may promote endothelial cell dysfunction, leading to excessive thrombin production and fibrinolysis inhibition [25]. At present, numerous studies have reported the potential development of the hypercoagulable state of COVID19: triggering a strong inflammatory response, leading to hypercoagulable state and thromboembolism [26-30]. In addition, hypoxemia is related to the increase in blood viscosity and the activation of hypoxia-related genes. Hypoxiarelated genes mediate coagulation and fibrinolysis, which is conducive to the occurrence of thrombotic events [31]. The COVID-19 often causes hypoxemia, especially severe pneumonia, which promotes the occurrence of embolic events. Acute inflammation caused by COVID-19 is prone to hypercoagulable state, which is due to the early molecular events caused by abnormal coagulation due to the increased concentration of pro-inflammatory cytokines and serum inflammatory factors (such as interleukin and C-reactive protein) [32-34]. Depending on the blood laboratory test indicators, patients with COVID-19 and cerebrovascular disease have severe inflammation and infection, and are in a hypercoagulable state. Significantly enhanced inflammation may be one of the reasons for the abnormal coagulation function in the initial stage, and it may also be one of the reasons for the onset of cerebrovascular disease [27]. In short, the mechanism of stroke caused by COVID-19 is currently unclear. At present, most opinions are still focused on the hypercoagulable state brought about by inflammation [7, 26, 27, 30, 32-36]. Severe Acute Respiratory Syndrome Coronavirus 2 directly infects endothelial cells, causing diffuse endothelial inflammation $[7,24,25,30,37,38]$. This may be the mechanism leading to ischemic stroke or cerebral hemorrhage. Other mechanisms linked to new coronary pneumonia in ischemic stroke include infection-induced hypercoagulable state, viral cardiomyopathy, and diffuse hyperinflammatory state. Therefore, in clinical practice, active control of inflammatory reactions and anticoagulation may be a powerful measure to preventing stroke caused by COVID19. Nonetheless, this relies on further powerful randomized controlled trials to verify.

It is also worth noting that the vast majority of ischemic stroke patients associated with COVID-19 in our study had common vascular risk factors such as hypertension, hyperlipidemia, and diabetes [39-45]. A study from Lebanon showed that a total of 284 cases were included with a mean age of 72 years, and 58\% male gender [46]. The most commonly identified risk factors were hypertension, dyslipidemia, and diabetes mellitus [42, 47-50]. We found that hypertension pooled prevalence was $66 \%$ (95\% CI 51-81\%; $p<0.01$ ), in keeping with other modern stroke cohorts [51]. In our study, hyperlipidemia occupies a prominent position. In Pawelczyk M et al. study, they reported that hyperlipidemia could promote the activation of platelets [52]. Active control of these high-risk factors, especially hyperlipidemia, is of positive significance for reducing ischemic stroke caused by COVID- 19 .

As we all know, gender is a significant factor in ischemic stroke [53-55]. Women differ from men in the distribution of risk factors and stroke subtype, stroke severity, and outcome. In the study of Arboix et al. [56], 2318 women and 2274 men with first-ever stroke were included. Vascular risk factors such as high blood pressure, atrial fibrillation, heart failure, valvular heart disease, and obesity are more common in women. Men are more likely to suffer from lacunar infarction $(21.5 \%$ vs. $16.2 \%, p=0.0003)$, while women are more likely to suffer from cardiogenic stroke (26\% vs. $15.6 \%, p=0.0001)$. It should be noted that cardioembolic infarctions are the most severe ischemic stroke subtype, with a high early mortality rate and degree of disability. Early outcome is worse in women with a higher in-hospital mortality, longer hospital stay and more disabled. However, in our research, we found that ischemic stroke caused by COVID19 is more likely to occur in men. The reason may be related to the gender imbalance in the detection of COVID-19 cases and fatality rates [57-59]. In addition, male patients may 
have more severe new coronary pneumonia and may lead to more severe inflammation [60]. Other form of strokes associated with other viral diseases was also learned in the research field. For examples, in the study of Helmuth, they found that men accounted for $67 \%$ in post-varicella arterial ischemic stroke [61]. This was consistent with our research that viral infection-related strokes are more likely to occur in men.

In our review, we also found that the proportion of cryptogenic stroke has increased. This may be linked to the unknown mechanism of ischemic stroke caused by COVID19. In addition, the frequency of acute strokes due to small vessel disease was much lower than the approximately $20 \%$ mentioned in most stroke databanks [62]. The cause of this phenomenon, however, was not clearly known. Perhaps it was related to the small amount of sample data we included in the study. Cerebral small vessel disease would be a crucial role in the field of stroke, especially lacunar stroke.

\section{Limitation}

Our study also has some limitations, most importantly, our research is limited by the fact that the selected literatures are all retrospective observational studies, and the different sample sizes of each study lead to inherent selection bias. Another limitation is that the variables we discussed are limited, such as some laboratory indicators, imaging characteristics, related treatment methods and prognosis are not included in the analysis of our article.

\section{Conclusions}

Ischemic stroke caused by COVID-19 has its own unique clinical features. Although common high-risk factors can also be observed, its importance may have changed. The major inflammatory storm of COVID-19 is more likely to occur in male patients. The increase in the proportion of cryptogenic stroke also made stroke related to COVID-19 complicated. Therefore, the risk of ischemic stroke must be taken into consideration when the patient was admitted with COVID-19. Patients may benefit from the early initiation of anti-inflammatory and anticoagulant therapy. However, further clinical trials are needed to be done for verified evidences.

Supplementary Information The online version contains supplementary material available at https://doi.org/10.1007/s00415-021-10837-7.

Author contributions WL made substantial contributions to conception and design of the study, analysis and interpretation of data, and to the drafting of the manuscript. XL made substantial contributions to the analysis and interpretation of the data and to the drafting of the manuscript. KB made substantial contributions to the conception and design of the study, analysis and interpretation of the data, and to the drafting of the manuscript. $\mathrm{CH}$ made substantial contributions to the conception and design of the study, and to drafting and revising the manuscript. All the authors have given final approval of the version of the manuscript to be published.

Funding There is no funding source.

Availability of data and materials Most of analyzed data were included in this manuscript and supplemental materials. The relevant data can also be obtained with the request from any qualified investigator for purposes of replicating procedures and results.

Code availability Not applicable.

\section{Declarations}

Conflicts of interest On behalf of all the authors, the corresponding author states that there is no conflict of interest.

Ethics approval Not applicable.

Consent to participate Not applicable.

Consent for publication Not applicable.

\section{References}

1. Wang D, Hu B, Hu C, Zhu F, Liu X, Zhang J, Wang B, Xiang $\mathrm{H}$, Cheng Z, Xiong Y et al (2020) Clinical characteristics of 138 hospitalized patients with 2019 novel coronavirus-infected pneumonia in Wuhan, China. JAMA 323(11):1061-1069

2. Mao L, Jin H, Wang M, Hu Y, Chen S, He Q, Chang J, Hong C, Zhou Y, Wang D et al (2020) Neurologic manifestations of hospitalized patients with coronavirus disease 2019 in Wuhan, China. JAMA Neurol 77(6)

3. Rothstein A, Oldridge O, Schwennesen H, Do D, Cucchiara BL (2020) Acute cerebrovascular events in hospitalized COVID-19 patients. Stroke 51(9):e219-e222

4. Lodigiani C, Iapichino G, Carenzo L, Cecconi M, Ferrazzi P, Sebastian T, Kucher N, Studt JD, Sacco C, Bertuzzi A et al (2020) Venous and arterial thromboembolic complications in COVID-19 patients admitted to an academic hospital in Milan, Italy. Thromb Res 191:9-14

5. Klok FA, Kruip M, van der Meer NJM, Arbous MS, Gommers D, Kant KM, Kaptein FHJ, van Paassen J, Stals MAM, Huisman MV et al (2020) Confirmation of the high cumulative incidence of thrombotic complications in critically ill ICU patients with COVID-19: an updated analysis. Thromb Res 191:148-150

6. Yaghi S, Ishida K, Torres J, Mac Grory B, Raz E, Humbert K, Henninger N, Trivedi T, Lillemoe K, Alam S et al (2020) SARSCoV-2 and stroke in a New York healthcare system. Stroke 51(7):2002-2011

7. Qin C, Zhou L, Hu Z, Zhang S, Yang S, Tao Y, Xie C, Ma K, Shang K, Wang W et al (2020) Dysregulation of immune response in patients with coronavirus 2019 (COVID-19) in Wuhan, China. Clin Infect Dis 71(15):762-768

8. Wu Y, Xu X, Chen Z, Duan J, Hashimoto K, Yang L, Liu C, Yang C (2020) Nervous system involvement after infection with COVID-19 and other coronaviruses. Brain Behav Immun $87: 18-22$ 
9. Steardo L, Steardo L Jr, Zorec R, Verkhratsky A (2020) Neuroinfection may contribute to pathophysiology and clinical manifestations of COVID-19. Acta Physiol (Oxf) 229(3):e13473

10. Xia H, Sriramula S, Chhabra KH, Lazartigues E (2013) Brain angiotensin-converting enzyme type 2 shedding contributes to the development of neurogenic hypertension. Circ Res 113(9):1087-1096

11. Fraga-Silva RA, Da Silva DG, Montecucco F, Mach F, Stergiopulos N, da Silva RF, Santos RAS (2012) The angiotensinconverting enzyme 2/angiotensin-(1-7)/Mas receptor axis: a potential target for treating thrombotic diseases. Thromb Haemost 108(6):1089-1096

12. Moher D, Liberati A, Tetzlaff J, Altman DG, Group P (2009) Preferred reporting items for systematic reviews and meta-analyses: the PRISMA statement. BMJ 339:b2535

13. Muka T, Glisic M, Milic J, Verhoog S, Bohlius J, Bramer W, Chowdhury R, Franco OH (2020) A 24-step guide on how to design, conduct, and successfully publish a systematic review and meta-analysis in medical research. Eur J Epidemiol 35(1):49-60

14. Stang A (2010) Critical evaluation of the Newcastle-Ottawa scale for the assessment of the quality of nonrandomized studies in meta-analyses. Eur J Epidemiol 25(9):603-605

15. Higgins JPT, Thompson SG, Deeks JJ, Altman DG (2003) Measuring inconsistency in meta-analyses. BMJ (Clinical Research ed) 327(7414):557-560

16. Behzadnia H, Omrani SN, Nozari-Golsefid H, Moslemi S, Alijani B, Reyhanian Z, Kaviani M, Heydari T, Andalib S (2020) Ischemic stroke and intracerebral hemorrhage in patients with COVID-19. Romanian J Neurol 19(3):166-170

17. Shahjouei S, Naderi S, Li J, Khan A, Chaudhary D, Farahmand G, Male S, Griessenauer C, Sabra M, Mondello S et al (2020) Risk of stroke in hospitalized SARS-CoV-2 infected patients: a multinational study. EBioMedicine 59:102939

18. John S, Hussain SI, Piechowski-Jozwiak B, Dibu J, Kesav P, Bayrlee A, Elkambergy H, John TLS, Roser F, Mifsud VA (2020) Clinical characteristics and admission patterns of stroke patients during the COVID 19 pandemic: a single center retrospective, observational study from the Abu Dhabi, United Arab Emirates. Clin Neurol Neurosurg 199:106227

19. Mendes A, Herrmann FR, Genton L, Serratrice C, Carrera E, Vargas MI, Gold G, Graf CE, Zekry D, Scheffler M (2021) Incidence, characteristics and clinical relevance of acute stroke in old patients hospitalized with COVID-19. BMC Geriatr 21(1):52

20 Adams HP Jr, Bendixen BH, Kappelle LJ, Biller J, Love BB, Gordon DL, Marsh EE 3rd (1993) Classification of subtype of acute ischemic stroke. Definitions for use in a multicenter clinical trial. TOAST. Trial of Org 10172 in Acute Stroke Treatment. Stroke 24(1):35-41

21. Chen PH, Gao S, Wang YJ, Xu AD, Li YS, Wang D (2012) Classifying ischemic stroke, from TOAST to CISS. CNS Neurosci Ther 18(6):452-456

22. Tan YK, Goh C, Leow AST, Tambyah PA, Ang A, Yap ES, Tu TM, Sharma VK, Yeo LLL, Chan BPL et al (2020) COVID-19 and ischemic stroke: a systematic review and meta-summary of the literature. J Thromb Thrombolysis 50(3):587-595

23. Grau AJ, Buggle F, Becher H, Zimmermann E, Spiel M, Fent T, Maiwald M, Werle E, Zorn M, Hengel H et al (1998) Recent bacterial and viral infection is a risk factor for cerebrovascular ischemia: clinical and biochemical studies. Neurology 50(1):196-203

24. Pranata R, Huang I, Lim MA, Wahjoepramono EJ, July J (2020) Impact of cerebrovascular and cardiovascular diseases on mortality and severity of COVID-19-systemati c review, meta-analysis, and meta-regression. J Stroke Cerebrovasc Dis 29(8):104949

25. Bibas M, Biava G, Antinori A (2011) HIV-associated venous thromboembolism. Mediterr J Hematol Infect Dis 3(1):e2011030
26. Divani AA, Andalib S, Di Napoli M, Lattanzi S, Hussain MS, Biller J, McCullough LD, Azarpazhooh MR, Seletska A, Mayer SA et al (2020) Coronavirus Disease 2019 and stroke: clinical manifestations and pathophysiological insights. J Stroke Cerebrovasc Dis 29(8): 104941

27. Li Y, Li M, Wang M, Zhou Y, Chang J, Xian Y, Wang D, Mao L, Jin H, Hu B (2020) Acute cerebrovascular disease following COVID-19: a single center, retrospective, observational study. Stroke Vasc Neurol 5(3):279-284

28. Wang W, Sun Q, Bao Y, Liang M, Meng Q, Chen H, Li J, Wang $\mathrm{H}$, Li H, Shi Y et al (2021) Analysis of risk factors for thromboembolic events in 88 patients with COVID-19 pneumonia in Wuhan, China: a retrospective descriptive report. Med Sci Monit 27:e929708

29. Han H, Yang L, Liu R, Liu F, Wu KL, Li J, Liu XH, Zhu CL (2020) Prominent changes in blood coagulation of patients with SARS-CoV-2 infection. Clin Chem Lab Med 58(7):1116-1120

30. Zhang Y, Xiao M, Zhang S, Xia P, Cao W, Jiang W, Chen H, Ding $\mathrm{X}$, Zhao H, Zhang H et al (2020) Coagulopathy and antiphospholipid antibodies in patients with Covid-19. N Engl J Med 382(17):e38

31. Gupta N, Zhao YY, Evans CE (2019) The stimulation of thrombosis by hypoxia. Thromb Res 181:77-83

32. Huang C, Wang Y, Li X, Ren L, Zhao J, Hu Y, Zhang L, Fan G, Xu J, Gu X et al (2020) Clinical features of patients infected with 2019 novel coronavirus in Wuhan, China. Lancet 395(10223):497-506

33. Olson G, Davis AM (2020) Diagnosis and treatment of adults with community-acquired pneumonia. JAMA 323(9):885-886

34. Metlay JP, Waterer GW, Long AC, Anzueto A, Brozek J, Crothers K, Cooley LA, Dean NC, Fine MJ, Flanders SA et al (2019) Diagnosis and treatment of adults with community-acquired pneumonia. An official clinical practice guideline of the American thoracic society and infectious diseases society of America. Am J Respir Crit Care Med 200(7):e45-e67

35. Ding P, Zhang S, Yu M, Feng Y, Long Q, Yang H, Li J, Wang M (2018) IL-17A promotes the formation of deep vein thrombosis in a mouse model. Int Immunopharmacol 57:132-138

36. Horvei LD, Grimnes G, Hindberg K, Mathiesen EB, Njølstad I, Wilsgaard T, Brox J, Braekkan SK, Hansen JB (2016) C-reactive protein, obesity, and the risk of arterial and venous thrombosis. J Thromb Haemost: JTH 14(8):1561-1571

37. Squizzato A, Gerdes VEA, Büller HR (2005) Effects of human cytomegalovirus infection on the coagulation system. Thromb Haemost 93(3):403-410

38. Uthman IW, Gharavi AE (2002) Viral infections and antiphospholipid antibodies. Semin Arthritis Rheum 31(4):256-263

39. Hui C, Patti L (2018) Stroke, Ischemic (StatPearls[Internet]). In., edn. eng

40. Gorgui J, Gorshkov M, Khan N, Daskalopoulou SS (2014) Hypertension as a risk factor for ischemic stroke in women. Can J Cardiol 30(7):774-782

41. Powers WJ (2020) Acute ischemic stroke. N Engl J Med 383(3):252-260

42. Diener H-C, Hankey GJ (2020) Primary and secondary prevention of ischemic stroke and cerebral hemorrhage: JACC focus seminar. J Am Coll Cardiol 75(15):1804-1818

43. Cipolla MJ, Liebeskind DS, Chan S-L (2018) The importance of comorbidities in ischemic stroke: impact of hypertension on the cerebral circulation. J Cereb Blood Flow Metab 38(12):2129-2149

44. Maïer B, Kubis N (2019) Hypertension and its impact on stroke recovery: from a vascular to a parenchymal overview. Neural Plast 2019:6843895

45. Oza R, Rundell K, Garcellano M (2017) Recurrent ischemic stroke: strategies for prevention. Am Fam Physician 96(7):436-440 
46. Malek EG, Elbejjani M, Abbas R, Abed Al Ahad M, Ismaeel H, Makki A (2020) TOAST classification and risk factors of ischemic stroke in Lebanon. Acta Neurol Scand 141(4):294-300

47. Pan Y, Elm JJ, Li H, Easton JD, Wang Y, Farrant M, Meng X, Kim AS, Zhao X, Meurer WJ et al (2019) Outcomes associated with clopidogrel-aspirin use in minor stroke or transient ischemic attack: a pooled analysis of clopidogrel in high-risk patients with acute non-disabling cerebrovascular events (CHANCE) and platelet-oriented inhibition in new TIA and minor ischemic stroke (POINT) trials. JAMA Neurol 76(12):1466-1473

48. Sun L, Clarke R, Bennett D, Guo Y, Walters RG, Hill M, Parish S, Millwood IY, Bian Z, Chen Y et al (2019) Causal associations of blood lipids with risk of ischemic stroke and intracerebral hemorrhage in Chinese adults. Nat Med 25(4):569-574

49. Li L, Yiin GS, Geraghty OC, Schulz UG, Kuker W, Mehta Z, Rothwell PM (2015) Incidence, outcome, risk factors, and long-term prognosis of cryptogenic transient ischaemic attack and ischaemic stroke: a population-based study. Lancet Neurol 14(9):903-913

50. Chen D-Y, See L-C, Liu J-R, Chuang C-K, Pang S-T, Hsieh IC, Wen M-S, Chen T-H, Lin Y-C, Liaw C-C et al (2017) Risk of cardiovascular ischemic events after surgical castration and gonadotropin-releasing hormone agonist therapy for prostate cancer: a nationwide cohort study. J Clin Oncol 35(32):3697-3705

51. Ornello R, Degan D, Tiseo C, Di Carmine C, Perciballi L, Pistoia F, Carolei A, Sacco S (2018) Distribution and temporal trends from 1993 to 2015 of ischemic stroke subtypes: a systematic review and meta-analysis. Stroke 49(4):814-819

52. Pawelczyk M, Kaczorowska B, Baj Z (2017) The impact of hyperglycemia and hyperlipidemia on plasma P-selectin and platelet markers after ischemic stroke. Arch Med Sci 13(5):1049-1056

53. Roy-O'Reilly M, McCullough LD (2018) Age and sex are critical factors in ischemic stroke pathology. Endocrinology 159(8):3120-3131
54. Guo J, Guan TJ, Liu YL, Chao BH, Wang LD (2019) Gender-specific factors of ischemic stroke among atrial fibrillation patients. Zhonghua Yu Fang Yi Xue Za Zhi 53(11):1136-1140

55. Hu J, Lin JH, Jiménez MC, Manson JE, Hankinson SE, Rexrode KM (2020) Plasma estradiol and testosterone levels and ischemic stroke in postmenopausal women. Stroke 51(4):1297-1300

56 Arboix A, Cartanya A, Lowak M, Garcia-Eroles L, Parra O, Oliveres M, Massons J (2014) Gender differences and woman-specific trends in acute stroke: results from a hospital-based registry (1986-2009). Clin Neurol Neurosurg 127:19-24

57. Guan WJ, Ni ZY, Hu Y, Liang WH, Ou CQ, He JX, Liu L, Shan H, Lei CL, Hui DSC et al (2020) Clinical characteristics of coronavirus disease 2019 in China. N Engl J Med 382(18):1708-1720

58. Zhao S, Cao P, Chong MKC, Gao D, Lou Y, Ran J, Wang K, Wang W, Yang L, He D et al (2020) COVID-19 and gender-specific difference: analysis of public surveillance data in Hong Kong and Shenzhen, China, from January 10 to February 15, 2020. Infect Control Hosp Epidemiol 41(6):750-751

59. Mo P, Xing Y, Xiao Y, Deng L, Zhao Q, Wang H, Xiong Y, Cheng Z, Gao S, Liang K et al (2020) Clinical characteristics of refractory COVID-19 pneumonia in Wuhan, China. Clin Infect Dis

60. Gebhard C, Regitz-Zagrosek V, Neuhauser HK, Morgan R, Klein SL (2020) Impact of sex and gender on COVID-19 outcomes in Europe. Biol Sex Differ 11(1):29

61. Helmuth IG, Mølbak K, Uldall PV, Poulsen A (2018) Post-varicella arterial ischemic stroke in Denmark 2010 to 2016. Pediatr Neurol 80:42-50

62 Arboix A, Blanco-Rojas L, Marti-Vilalta JL (2014) Advancements in understanding the mechanisms of symptomatic lacunar ischemic stroke: translation of knowledge to prevention strategies. Expert Rev Neurother 14(3):261-276 